Supporting information

\title{
Synthesis and Properties of Novel Dithienothiasiline Derivatives
}

Kwang-Hoi Lee, Joji Ohshita,* and Atsutaka Kunai*

Department of Applied Chemistry, Graduate School of Engineering, Hiroshima University,

Higashi-Hiroshima 739-8527, Japan

Table 1. Atomic coordinates and $\mathrm{B}_{\mathrm{iso}} / \mathrm{B}_{\mathrm{eq}}$

$\begin{array}{lcccl}\text { atom } & \mathrm{x} & \mathrm{y} & \mathrm{z} & \mathrm{B} \text { eq } \\ \mathrm{S}(1) & 0.3109(1) & 0.4036(1) & 0.4638(1) & 2.20(5) \\ \mathrm{S}(2) & 0.1066(1) & 0.5069(1) & 0.4434(1) & 2.17(5) \\ \mathrm{S}(3) & -0.1354(1) & 0.5578(1) & 0.3819(1) & 2.19(5) \\ \mathrm{S}(4) & 0.3390(1) & -0.1462(1) & 0.3861(1) & 2.28(5) \\ \mathrm{S}(5) & 0.5942(2) & -0.0653(1) & 0.4250(1) & 2.41(5) \\ \mathrm{S}(6) & 0.8147(1) & 0.0494(1) & 0.4382(1) & 2.15(4) \\ \mathrm{Si}(1) & 0.4916(2) & 0.2728(1) & 0.4058(1) & 2.40(5) \\ \mathrm{Si}(2) & -0.3661(2) & 0.5660(1) & 0.2436(1) & 2.43(5) \\ \mathrm{Si}(3) & 0.0799(2) & 0.4042(1) & 0.2179(1) & 2.10(5) \\ \mathrm{Si}(4) & 0.0691(2) & -0.2192(1) & 0.2760(1) & 2.17(5) \\ \mathrm{Si}(5) & 0.9835(2) & 0.1578(1) & 0.3746(1) & 2.35(5) \\ \mathrm{Si}(6) & 0.5050(2) & -0.0745(1) & 0.2074(1) & 1.88(5) \\ \mathrm{C}(1) & 0.3612(5) & 0.3373(4) & 0.3808(4) & 1.9(2) \\ \mathrm{C}(2) & 0.2917(5) & 0.3365(4) & 0.3036(4) & 1.9(2) \\ \mathrm{C}(3) & 0.1964(6) & 0.3902(4) & 0.3086(4) & 1.8(2) \\ \mathrm{C}(4) & 0.1981(5) & 0.4308(4) & 0.3933(4) & 1.6(2) \\ \mathrm{C}(5) & -0.0135(5) & 0.5048(4) & 0.3561(4) & 2.0(2) \\ \mathrm{C}(6) & -0.0282(5) & 0.4712(4) & 0.2698(4) & 1.9(2) \\ \mathrm{C}(7) & -0.1409(5) & 0.4935(5) & 0.2252(4) & 2.1(2) \\ \mathrm{C}(8) & -0.2124(6) & 0.5397(5) & 0.2759(4) & 2.2(2) \\ \mathrm{C}(9) & 0.1521(6) & 0.4659(5) & 0.1623(5) & 2.2(2) \\ \mathrm{C}(10) & 0.1211(6) & 0.4440(5) & 0.0733(5) & 2.8(2) \\ \mathrm{C}(11) & 0.1706(7) & 0.4962(6) & 0.0350(5) & 3.7(2) \\ \mathrm{C}(12) & 0.2535(7) & 0.5734(6) & 0.0866(5) & 3.6(2) \\ \mathrm{C}(13) & 0.2861(6) & 0.5968(5) & 0.1738(5) & 2.9(2) \\ \mathrm{C}(14) & 0.2378(6) & 0.5430(5) & 0.2115(5) & 2.5(2) \\ \mathrm{C}(15) & -0.0071(6) & 0.2906(5) & 0.1404(5) & 2.7(2) \\ \mathrm{C}(16) & 0.0345(8) & 0.2327(6) & 0.0759(5) & 4.0(2) \\ \mathrm{C}(17) & -0.0253(9) & 0.1473(7) & 0.0233(7) & 6.4(3) \\ \mathrm{C}(18) & -0.132(1) & 0.1203(7) & 0.0342(6) & 6.9(3) \\ \mathrm{C}(19) & -0.1820(7) & 0.1715(6) & 0.0932(7) & 5.4(3)\end{array}$




$\begin{array}{lrlll}\mathrm{C}(20) & -0.1195(7) & 0.2592(5) & 0.1490(5) & 3.3(2) \\ \mathrm{C}(21) & 0.5908(5) & 0.3435(5) & 0.5143(4) & 3.7(2) \\ \mathrm{C}(22) & 0.5679(6) & 0.2567(5) & 0.3210(5) & 3.9(2) \\ \mathrm{C}(23) & 0.4269(6) & 0.1659(4) & 0.4100(4) & 2.9(2) \\ \mathrm{C}(24) & -0.3662(6) & 0.6820(5) & 0.3150(5) & 4.2(2) \\ \mathrm{C}(25) & -0.4018(5) & 0.5573(5) & 0.1301(4) & 3.0(2)\end{array}$

$\mathrm{B}_{\mathrm{eq}}=8 / 3 \pi^{2}\left(\mathrm{U}_{11}\left(\mathrm{aa}^{*}\right)^{2}+\mathrm{U}_{22}\left(\mathrm{bb}^{*}\right)^{2}+\mathrm{U}_{33}(\mathrm{cc} *)^{2}+2 \mathrm{U}_{12}\left(\mathrm{aa}^{*} \mathrm{bb} *\right) \cos \gamma+\right.$ $\left.2 \mathrm{U}_{13}\left(\mathrm{aa}^{*} \mathrm{cc} *\right) \cos \beta+2 \mathrm{U}_{23}\left(\mathrm{bb}^{*} \mathrm{cc} *\right) \cos \alpha\right)$

Table 2. Anisotropic Displacement Parameters

\begin{tabular}{|c|c|c|c|c|c|c|}
\hline atom & $\mathrm{U}_{11}$ & $\mathrm{U}_{22}$ & U33 & $\mathrm{U}_{12}$ & $\mathrm{U}_{13}$ & $\mathrm{U}_{23}$ \\
\hline$S(1)$ & $0.026(1)$ & $0.033(1)$ & $0.030(1)$ & $0.0132(9)$ & $0.0071(9)$ & $0.016(1)$ \\
\hline$S(2)$ & $0.026(1)$ & $0.032(1)$ & $0.028(1)$ & $0.0113(9)$ & $0.0077(9)$ & $0.013(1)$ \\
\hline$S(3)$ & $0.026(1)$ & $0.032(1)$ & $0.032(1)$ & $0.0114(9)$ & $0.0109(9)$ & $0.017(1)$ \\
\hline$S(4)$ & $0.026(1)$ & $0.036(1)$ & $0.028(1)$ & $0.0002(9)$ & $0.0088(9)$ & $0.016(1)$ \\
\hline$S(5)$ & $0.028(1)$ & $0.039(1)$ & $0.026(1)$ & $-0.0029(9)$ & $0.0040(9)$ & $0.018(1)$ \\
\hline$S(6)$ & $0.025(1)$ & $0.032(1)$ & $0.025(1)$ & $0.0004(9)$ & $0.0055(9)$ & $0.011(1)$ \\
\hline $\operatorname{Si}(1)$ & $0.027(1)$ & $0.032(1)$ & $0.039(1)$ & $0.012(1)$ & $0.010(1)$ & $0.019(1)$ \\
\hline $\operatorname{Si}(2)$ & $0.026(1)$ & $0.032(1)$ & $0.037(1)$ & $0.013(1)$ & $0.006(1)$ & $0.016(1)$ \\
\hline $\operatorname{Si}(3)$ & $0.027(1)$ & $0.027(1)$ & $0.028(1)$ & $0.010(1)$ & $0.009(1)$ & $0.012(1)$ \\
\hline $\mathrm{Si}(4)$ & $0.024(1)$ & $0.029(1)$ & $0.032(1)$ & $0.004(1)$ & $0.007(1)$ & $0.015(1)$ \\
\hline $\operatorname{Si}(5)$ & $0.028(1)$ & $0.029(1)$ & $0.035(1)$ & $0.003(1)$ & $0.011(1)$ & $0.013(1)$ \\
\hline $\operatorname{Si}(6)$ & $0.026(1)$ & $0.025(1)$ & $0.024(1)$ & $0.004(1)$ & $0.0077(9)$ & $0.012(1)$ \\
\hline $\mathrm{C}(1)$ & $0.022(4)$ & $0.021(5)$ & $0.040(5)$ & $0.015(3)$ & $0.012(4)$ & $0.019(4)$ \\
\hline $\mathrm{C}(2)$ & $0.026(4)$ & $0.022(4)$ & $0.030(5)$ & $0.012(3)$ & $0.011(3)$ & $0.012(4)$ \\
\hline $\mathrm{C}(3)$ & $0.024(4)$ & $0.021(4)$ & $0.028(4)$ & $0.002(3)$ & $0.009(3)$ & $0.013(4)$ \\
\hline $\mathrm{C}(4)$ & $0.014(3)$ & $0.019(4)$ & $0.034(4)$ & $0.005(3)$ & $0.008(3)$ & $0.013(4)$ \\
\hline$C(5)$ & $0.019(4)$ & $0.023(5)$ & $0.036(5)$ & $0.002(3)$ & $0.010(3)$ & $0.014(4)$ \\
\hline$C(6)$ & $0.024(4)$ & $0.023(5)$ & $0.028(4)$ & $0.002(3)$ & $0.006(3)$ & $0.016(4)$ \\
\hline$C(7)$ & $0.026(4)$ & $0.034(5)$ & $0.024(4)$ & $0.001(4)$ & $0.002(3)$ & $0.017(4)$ \\
\hline $\mathrm{C}(8)$ & $0.028(4)$ & $0.027(5)$ & $0.034(5)$ & $0.004(4)$ & $0.010(4)$ & $0.015(4)$ \\
\hline $\mathrm{C}(9)$ & $0.031(4)$ & $0.030(5)$ & $0.028(5)$ & $0.017(4)$ & $0.010(4)$ & $0.013(4)$ \\
\hline$C(10)$ & $0.031(4)$ & $0.043(6)$ & $0.043(5)$ & $0.013(4)$ & $0.020(4)$ & $0.021(5)$ \\
\hline $\mathrm{C}(11)$ & $0.054(6)$ & $0.062(7)$ & $0.034(6)$ & $0.019(5)$ & $0.019(5)$ & $0.024(5)$ \\
\hline $\mathrm{C}(12)$ & $0.043(5)$ & $0.059(7)$ & $0.062(6)$ & $0.022(5)$ & $0.031(5)$ & $0.042(5)$ \\
\hline$C(13)$ & $0.024(4)$ & $0.038(6)$ & $0.048(6)$ & $0.009(4)$ & $0.008(4)$ & $0.017(5)$ \\
\hline $\mathrm{C}(14)$ & $0.027(4)$ & $0.039(5)$ & $0.036(5)$ & $0.012(4)$ & $0.012(4)$ & $0.016(4)$ \\
\hline$C(15)$ & $0.039(5)$ & $0.031(5)$ & $0.031(5)$ & $0.013(4)$ & $-0.005(4)$ & $0.019(4)$ \\
\hline$C(16)$ & $0.064(6)$ & $0.037(6)$ & $0.039(6)$ & $0.024(5)$ & $0.006(5)$ & $0.004(5)$ \\
\hline$C(17)$ & $0.093(9)$ & $0.056(9)$ & $0.069(8)$ & $0.027(7)$ & $-0.011(7)$ & $0.011(7)$ \\
\hline $\mathrm{C}(18)$ & $0.14(1)$ & $0.035(7)$ & $0.035(7)$ & $0.049(8)$ & $-0.033(8)$ & $-0.013(5)$ \\
\hline$C(19)$ & $0.043(6)$ & $0.051(7)$ & $0.096(9)$ & $-0.025(5)$ & $-0.035(6)$ & $0.053(7)$ \\
\hline$C(20)$ & $0.049(5)$ & $0.038(6)$ & $0.041(6)$ & $0.017(4)$ & $0.001(4)$ & $0.023(5)$ \\
\hline$C(21)$ & $0.028(4)$ & $0.032(5)$ & $0.070(6)$ & $0.002(4)$ & $-0.008(4)$ & $0.018(5)$ \\
\hline $\mathrm{C}(22)$ & $0.041(5)$ & $0.059(7)$ & $0.070(6)$ & $0.022(5)$ & $0.023(5)$ & $0.040(5)$ \\
\hline
\end{tabular}




$\begin{array}{lllllll}\mathrm{C}(23) & 0.042(5) & 0.033(5) & 0.041(5) & 0.017(4) & 0.012(4) & 0.017(4) \\ \mathrm{C}(24) & 0.032(5) & 0.054(6) & 0.058(6) & 0.029(4) & -0.004(4) & 0.007(5) \\ \mathrm{C}(25) & 0.034(4) & 0.043(6) & 0.045(5) & 0.018(4) & 0.009(4) & 0.023(5)\end{array}$

The general temperature factor expression: $\exp \left(-2 \pi^{2}\left(\mathrm{a}^{* 2} \mathrm{U} 11 \mathrm{~h}^{2}+\mathrm{b}^{* 2} \mathrm{U}_{22} \mathrm{k}^{2}+\mathrm{c}^{* 2} \mathrm{U}_{33} \mathrm{I}^{2}+\right.\right.$ $\left.\left.2 \mathrm{a} * \mathrm{~b} * \mathrm{U}_{12} \mathrm{hk}+2 \mathrm{a} * \mathrm{c} * \mathrm{U}_{13} \mathrm{hl}+2 \mathrm{~b} * \mathrm{c} * \mathrm{U}_{23} \mathrm{kl}\right)\right)$

Table 3. Bond lengths $(\AA)$

$\begin{array}{llllll}\text { atom } & \text { atom } & \text { distance } & \text { atom } & \text { atom } & \text { distance } \\ \mathrm{S}(1) & \mathrm{C}(1) & 1.711(7) & \mathrm{S}(1) & \mathrm{C}(4) & 1.715(6) \\ \mathrm{S}(2) & \mathrm{C}(4) & 1.780(6) & \mathrm{S}(2) & \mathrm{C}(5) & 1.753(6) \\ \mathrm{S}(3) & \mathrm{C}(5) & 1.737(6) & \mathrm{S}(3) & \mathrm{C}(8) & 1.730(7) \\ \mathrm{Si}(1) & \mathrm{C}(1) & 1.894(6) & \mathrm{Si}(1) & \mathrm{C}(21) & 1.855(7) \\ \mathrm{Si}(1) & \mathrm{C}(22) & 1.824(6) & \mathrm{Si}(1) & \mathrm{C}(23) & 1.841(6) \\ \mathrm{Si}(2) & \mathrm{C}(8) & 1.822(6) & \mathrm{Si}(2) & \mathrm{C}(24) & 1.840(7) \\ \mathrm{Si}(2) & \mathrm{C}(25) & 1.840(6) & \mathrm{Si}(2) & \mathrm{C}(26) & 1.867(6) \\ \mathrm{Si}(3) & \mathrm{C}(3) & 1.865(6) & \mathrm{Si}(3) & \mathrm{C}(6) & 1.869(7) \\ \mathrm{Si}(3) & \mathrm{C}(9) & 1.857(7) & \mathrm{Si}(3) & \mathrm{C}(15) & 1.888(8) \\ \mathrm{C}(1) & \mathrm{C}(2) & 1.359(8) & \mathrm{C}(2) & \mathrm{C}(3) & 1.419(8) \\ \mathrm{C}(3) & \mathrm{C}(4) & 1.372(8) & \mathrm{C}(5) & \mathrm{C}(6) & 1.358(8) \\ \mathrm{C}(6) & \mathrm{C}(7) & 1.453(8) & \mathrm{C}(7) & \mathrm{C}(8) & 1.396(8) \\ \mathrm{C}(9) & \mathrm{C}(10) & 1.393(8) & \mathrm{C}(9) & \mathrm{C}(14) & 1.395(9) \\ \mathrm{C}(10) & \mathrm{C}(11) & 1.386(9) & \mathrm{C}(11) & \mathrm{C}(12) & 1.39(1) \\ \mathrm{C}(12) & \mathrm{C}(13) & 1.361(9) & \mathrm{C}(13) & \mathrm{C}(14) & 1.390(8) \\ \mathrm{C}(15) & \mathrm{C}(16) & 1.386(9) & \mathrm{C}(15) & \mathrm{C}(20) & 1.410(9) \\ \mathrm{C}(16) & \mathrm{C}(17) & 1.38(1) & \mathrm{C}(17) & \mathrm{C}(18) & 1.34(1) \\ \mathrm{C}(18) & \mathrm{C}(19) & 1.34(1) & \mathrm{C}(19) & \mathrm{C}(20) & 1.43(1)\end{array}$

Table 4. Bond angles (O)

$\begin{array}{llllllll}\text { atom } & \text { atom } & \text { atom } & \text { angle } & \text { atom } & \text { atom } & \text { atom } & \text { angle } \\ \mathrm{C}(1) & \mathrm{S}(1) & \mathrm{C}(4) & 91.2(3) & \mathrm{C}(4) & \mathrm{S}(2) & \mathrm{C}(5) & 103.2(3) \\ \mathrm{C}(5) & \mathrm{S}(3) & \mathrm{C}(8) & 94.3(3) & \mathrm{C}(27) & \mathrm{S}(4) & \mathrm{C}(30) & 93.2(3) \\ \mathrm{C}(30) & \mathrm{S}(5) & \mathrm{C}(31) & 104.4(3) & \mathrm{C}(31) & \mathrm{S}(6) & \mathrm{C}(34) & 93.2(3) \\ \mathrm{C}(1) & \mathrm{Si}(1) & \mathrm{C}(21) & 106.3(3) & \mathrm{C}(1) & \mathrm{Si}(1) & \mathrm{C}(22) & 106.7(3) \\ \mathrm{C}(21) & \mathrm{Si}(1) & \mathrm{C}(22) & 113.2(3) & \mathrm{C}(1) & \mathrm{Si}(1) & \mathrm{C}(23) & 107.8(3) \\ \mathrm{C}(21) & \mathrm{Si}(1) & \mathrm{C}(23) & 109.1(3) & \mathrm{C}(22) & \mathrm{Si}(1) & \mathrm{C}(23) & 113.3(3) \\ \mathrm{C}(8) & \mathrm{Si}(2) & \mathrm{C}(24) & 106.6(3) & \mathrm{C}(8) & \mathrm{Si}(2) & \mathrm{C}(25) & 108.8(3) \\ \mathrm{C}(24) & \mathrm{Si}(2) & \mathrm{C}(25) & 111.3(3) & \mathrm{C}(8) & \mathrm{Si}(2) & \mathrm{C}(26) & 105.5(3) \\ \mathrm{C}(24) & \mathrm{Si}(2) & \mathrm{C}(26) & 111.6(3) & \mathrm{C}(25) & \mathrm{Si}(2) & \mathrm{C}(26) & 112.6(3) \\ \mathrm{C}(3) & \mathrm{Si}(3) & \mathrm{C}(6) & 104.8(3) & \mathrm{C}(3) & \mathrm{Si}(3) & \mathrm{C}(9) & 110.8(3) \\ \mathrm{C}(6) & \mathrm{Si}(3) & \mathrm{C}(9) & 110.8(3) & \mathrm{C}(3) & \mathrm{Si}(3) & \mathrm{C}(15) & 110.5(3) \\ \mathrm{C}(6) & \mathrm{Si}(3) & \mathrm{C}(15) & 108.4(3) & \mathrm{C}(9) & \mathrm{Si}(3) & \mathrm{C}(15) & 111.4(3) \\ \mathrm{S}(1) & \mathrm{C}(1) & \mathrm{Si}(1) & 118.8(4) & \mathrm{S}(1) & \mathrm{C}(1) & \mathrm{C}(2) & 111.1(5) \\ \mathrm{Si}(1) & \mathrm{C}(1) & \mathrm{C}(2) & 130.0(5) & \mathrm{C}(1) & \mathrm{C}(2) & \mathrm{C}(3) & 114.9(6) \\ \mathrm{Si}(3) & \mathrm{C}(3) & \mathrm{C}(2) & 127.8(5) & \mathrm{Si}(3) & \mathrm{C}(3) & \mathrm{C}(4) & 122.8(5) \\ & & & & & & & \end{array}$




$\begin{array}{llllllll}\mathrm{C}(2) & \mathrm{C}(3) & \mathrm{C}(4) & 109.4(6) & \mathrm{S}(1) & \mathrm{C}(4) & \mathrm{S}(2) & 114.4(4) \\ \mathrm{S}(1) & \mathrm{C}(4) & \mathrm{C}(3) & 113.5(5) & \mathrm{S}(2) & \mathrm{C}(4) & \mathrm{C}(3) & 132.1(5) \\ \mathrm{S}(2) & \mathrm{C}(5) & \mathrm{S}(3) & 116.2(4) & \mathrm{S}(2) & \mathrm{C}(5) & \mathrm{C}(6) & 131.5(5) \\ \mathrm{S}(3) & \mathrm{C}(5) & \mathrm{C}(6) & 112.3(5) & \mathrm{Si}(3) & \mathrm{C}(6) & \mathrm{C}(5) & 124.3(5) \\ \mathrm{Si}(3) & \mathrm{C}(6) & \mathrm{C}(7) & 126.0(5) & \mathrm{C}(5) & \mathrm{C}(6) & \mathrm{C}(7) & 109.7(6) \\ \mathrm{C}(6) & \mathrm{C}(7) & \mathrm{C}(8) & 116.9(6) & \mathrm{S}(3) & \mathrm{C}(8) & \mathrm{Si}(2) & 123.1(4) \\ \mathrm{S}(3) & \mathrm{C}(8) & \mathrm{C}(7) & 106.8(5) & \mathrm{Si}(2) & \mathrm{C}(8) & \mathrm{C}(7) & 129.7(5) \\ \mathrm{Si}(3) & \mathrm{C}(9) & \mathrm{C}(10) & 124.4(6) & \mathrm{Si}(3) & \mathrm{C}(9) & \mathrm{C}(14) & 119.1(5) \\ \mathrm{C}(10) & \mathrm{C}(9) & \mathrm{C}(14) & 116.3(7) & \mathrm{C}(9) & \mathrm{C}(10) & \mathrm{C}(11) & 122.3(7) \\ \mathrm{C}(10) & \mathrm{C}(11) & \mathrm{C}(12) & 119.3(8) & \mathrm{C}(11) & \mathrm{C}(12) & \mathrm{C}(13) & 119.9(7) \\ \mathrm{C}(12) & \mathrm{C}(13) & \mathrm{C}(14) & 120.4(7) & \mathrm{C}(9) & \mathrm{C}(14) & \mathrm{C}(13) & 121.7(7) \\ \mathrm{Si}(3) & \mathrm{C}(15) & \mathrm{C}(16) & 124.0(6) & \mathrm{Si}(3) & \mathrm{C}(15) & \mathrm{C}(20) & 119.5(6) \\ \mathrm{C}(16) & \mathrm{C}(15) & \mathrm{C}(20) & 116.5(8) & \mathrm{C}(15) & \mathrm{C}(16) & \mathrm{C}(17) & 123.8(9) \\ \mathrm{C}(16) & \mathrm{C}(17) & \mathrm{C}(18) & 117.6(1) & \mathrm{C}(17) & \mathrm{C}(18) & \mathrm{C}(19) & 123.8(1) \\ \mathrm{C}(18) & \mathrm{C}(19) & \mathrm{C}(20) & 119.0(9) & \mathrm{C}(15) & \mathrm{C}(20) & \mathrm{C}(19) & 119.4(8)\end{array}$




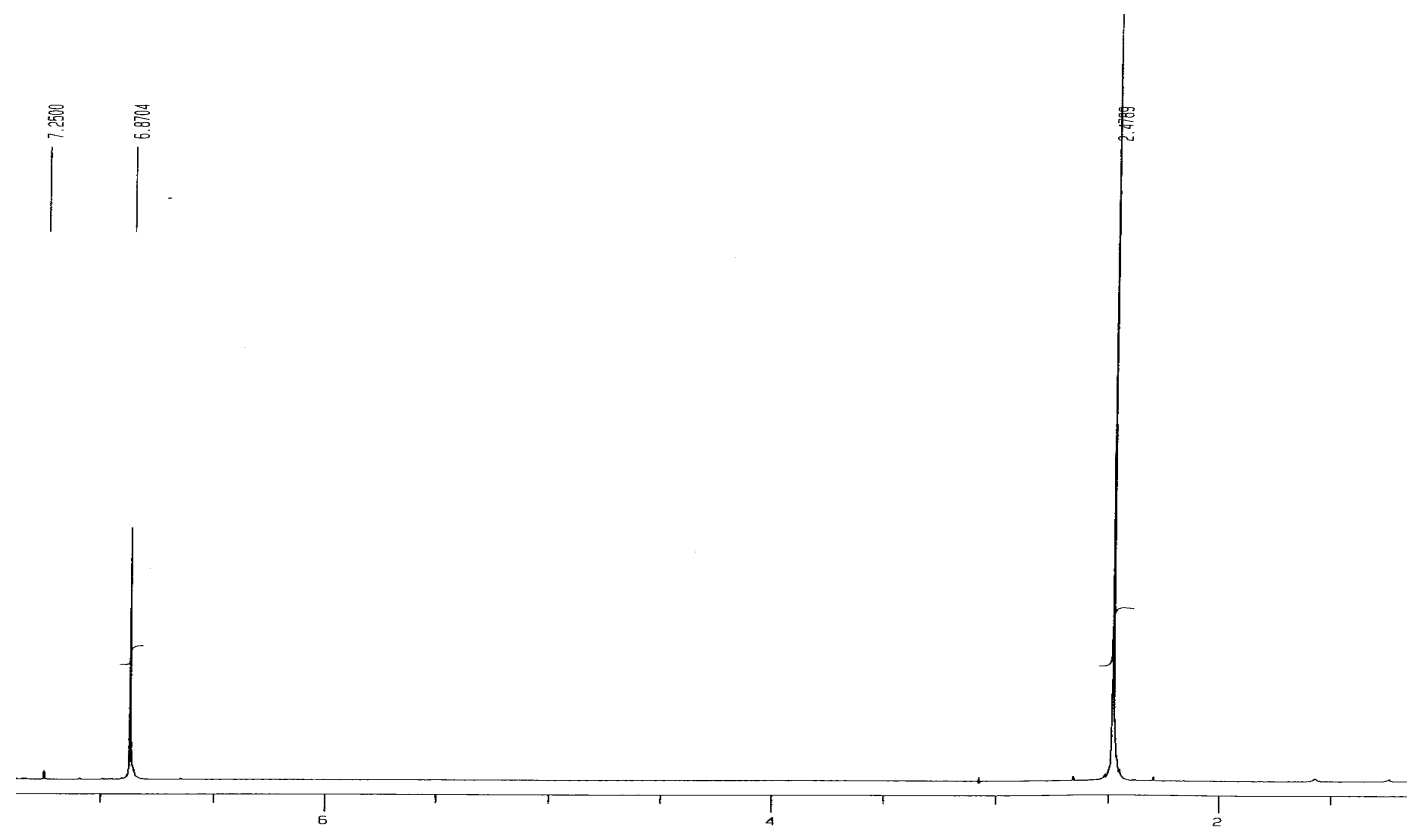

(a)

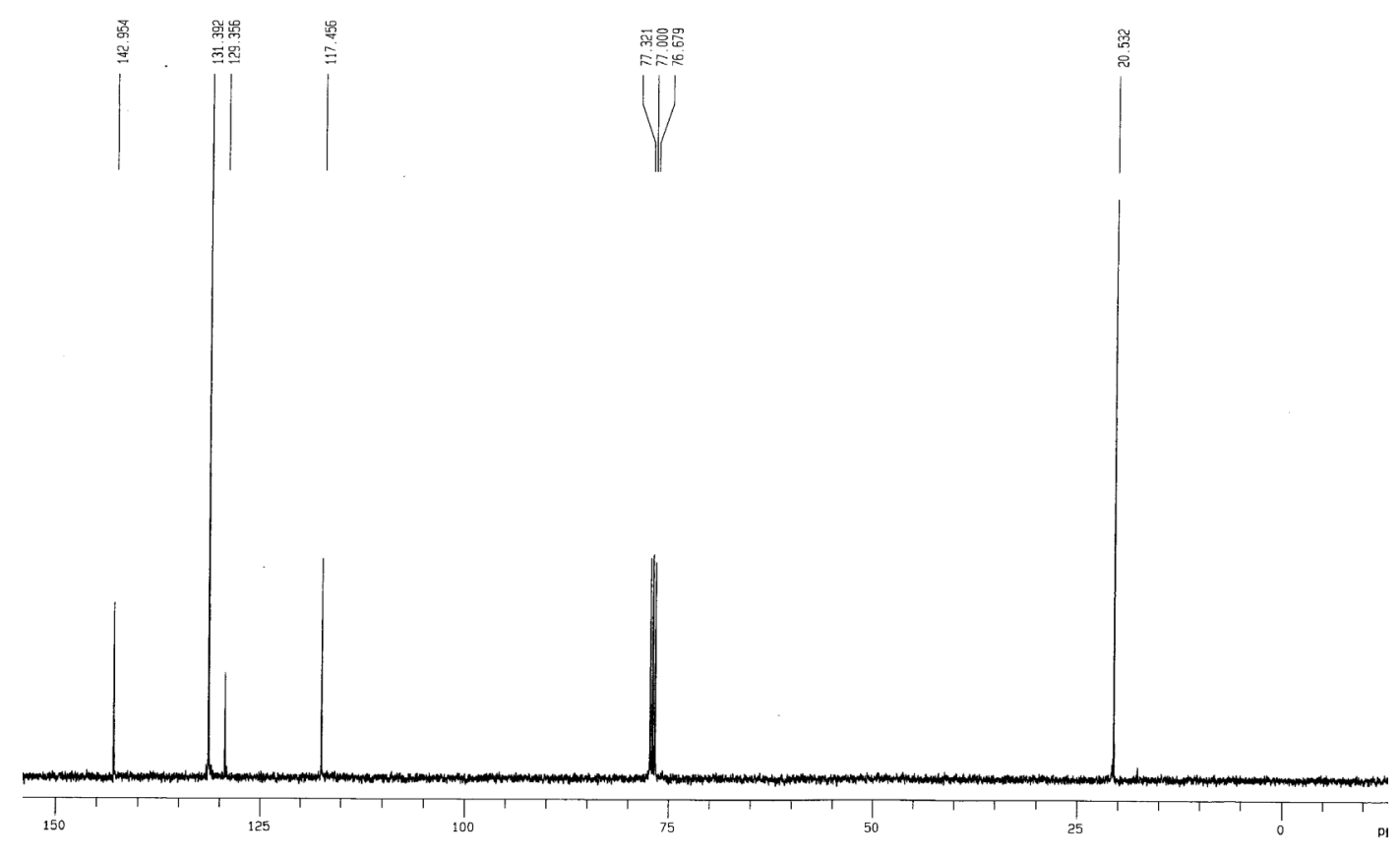

(b)

Figure 1. ${ }^{1} \mathrm{H}$ (a) and ${ }^{13} \mathrm{C}$ NMR spectra (b) of compound 2. 


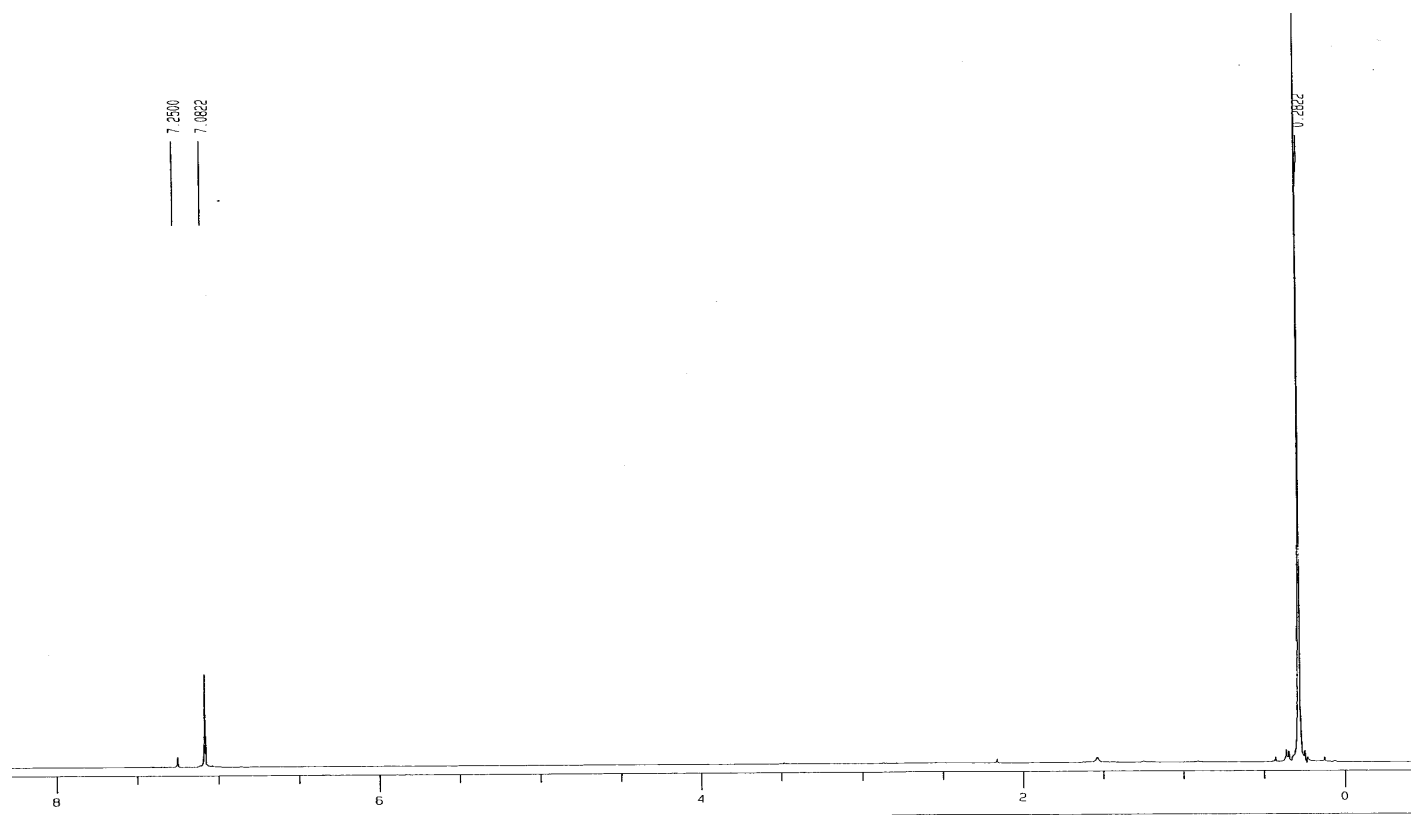

(a)

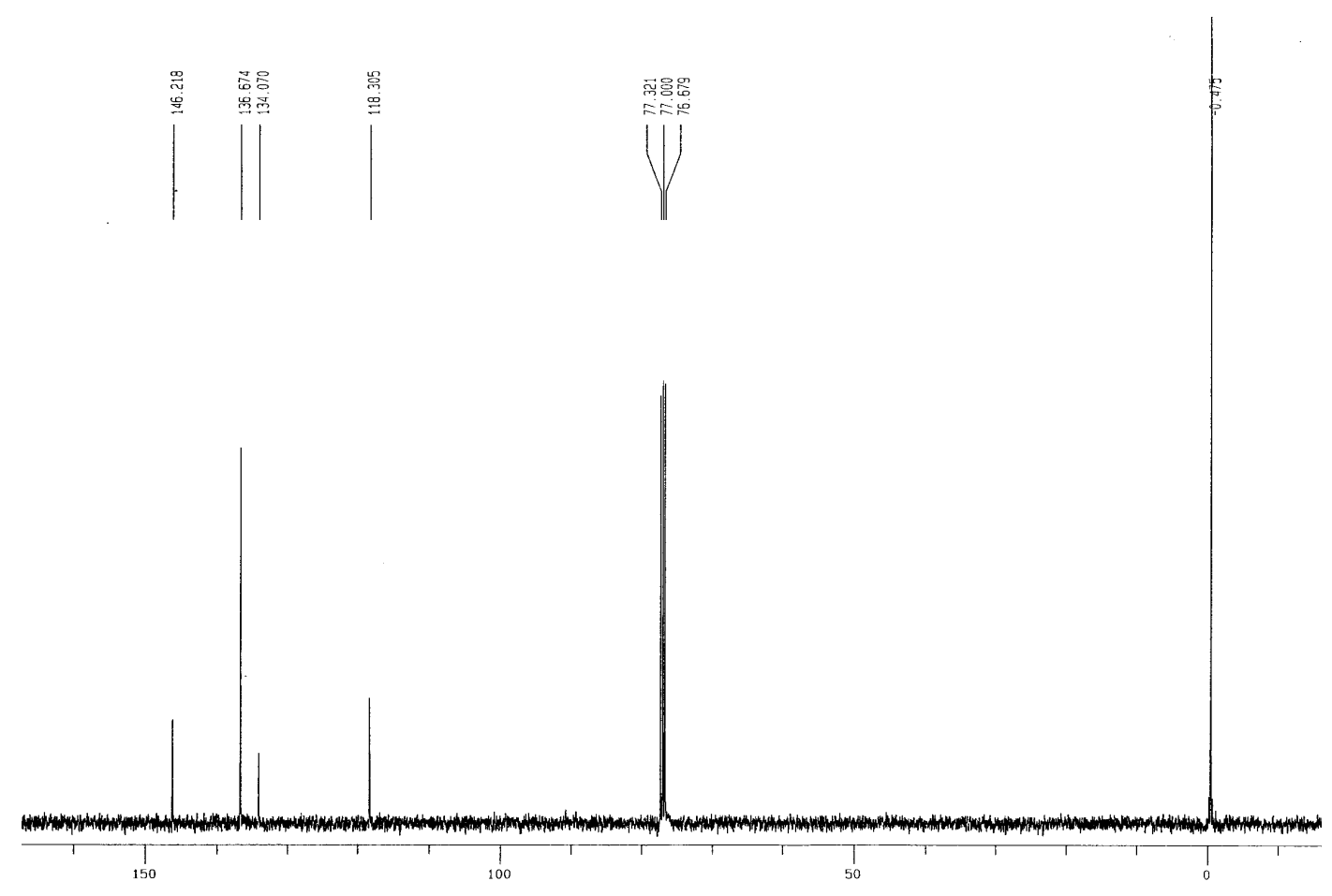

(b)

Figure 2. ${ }^{1} \mathrm{H}$ (a) and ${ }^{13} \mathrm{C}$ NMR spectra (b) of compound 3. 


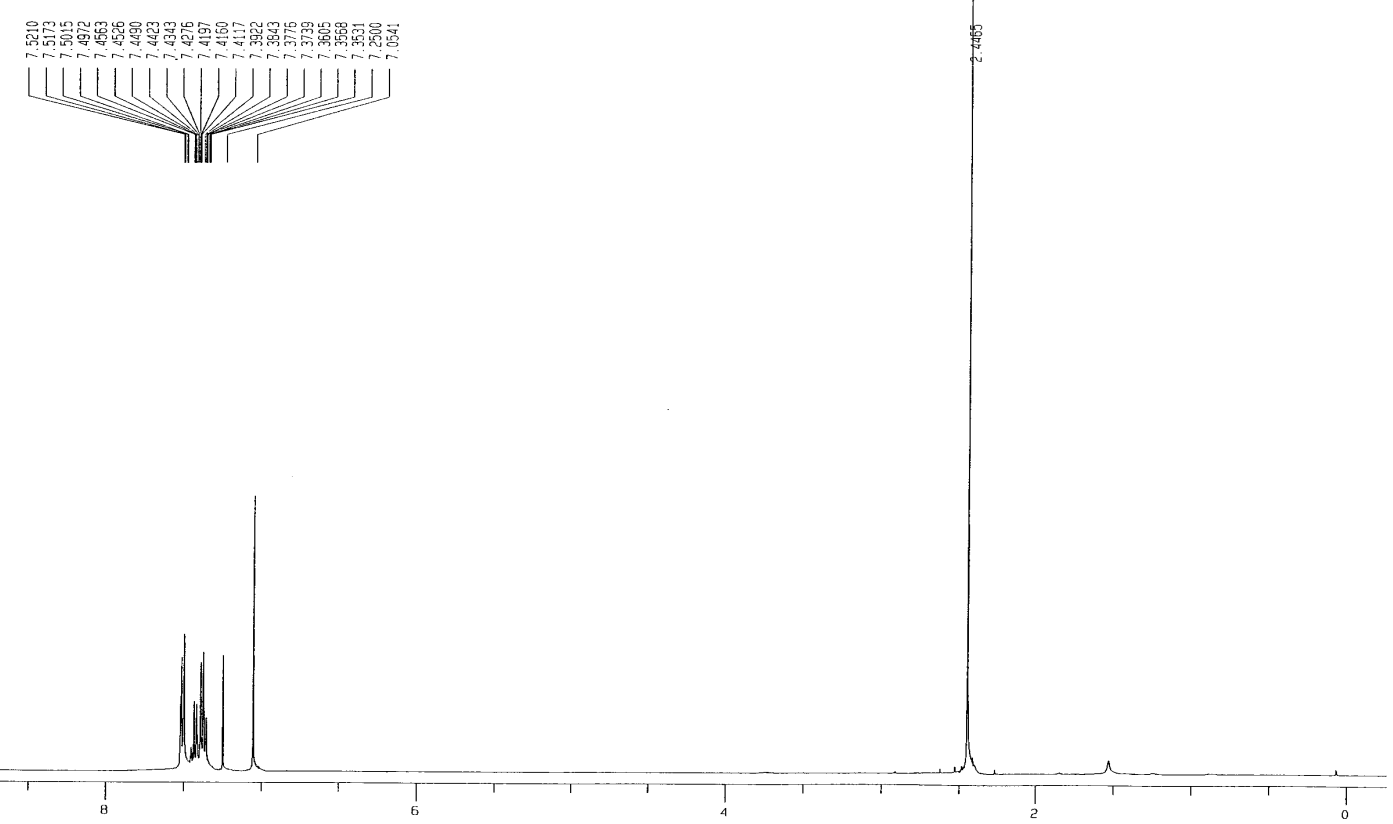

(a)

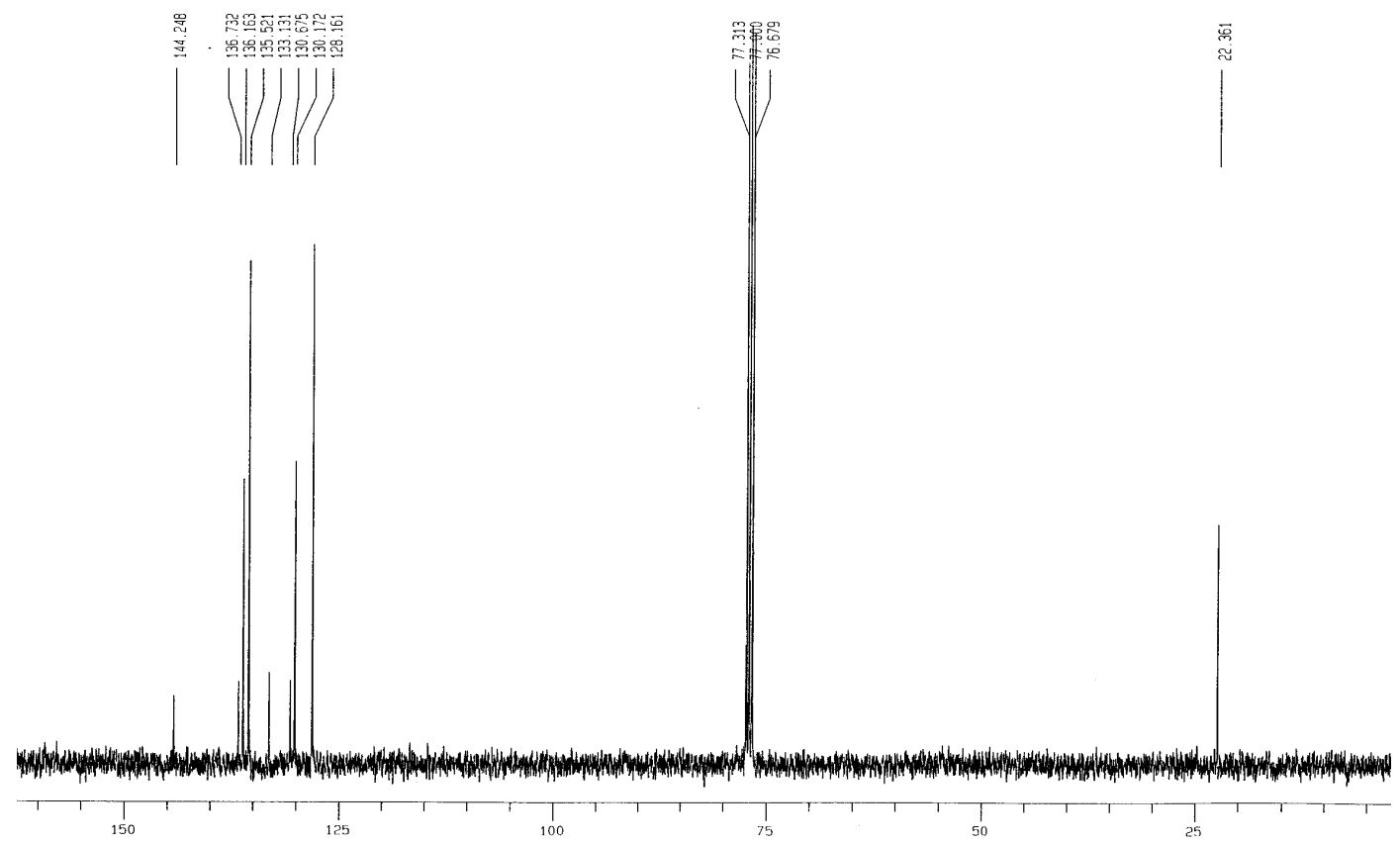

(b)

Figure 3. ${ }^{1} \mathrm{H}$ (a) and ${ }^{13} \mathrm{C}$ NMR spectra (b) of compound 4. 


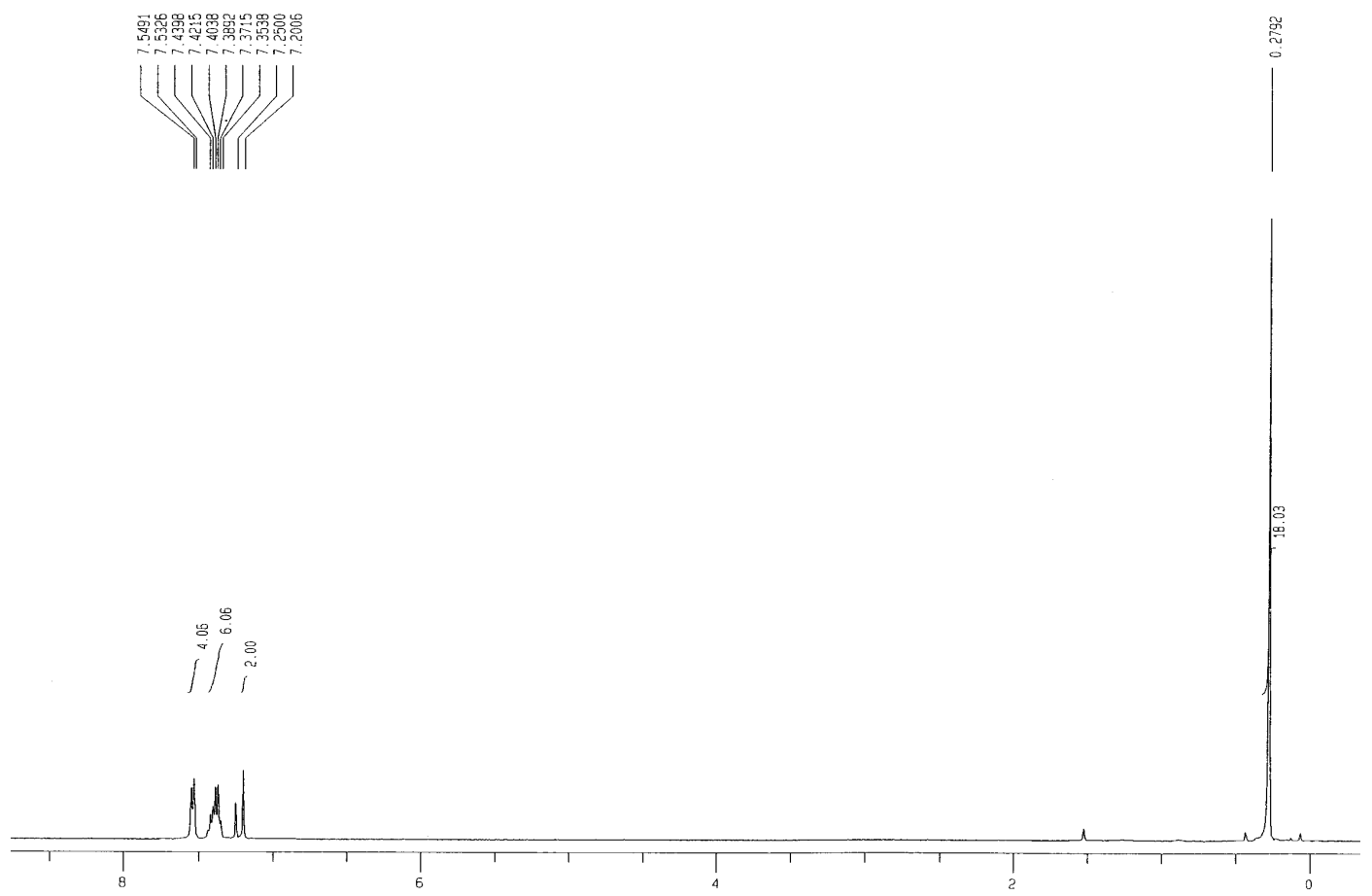

(a)

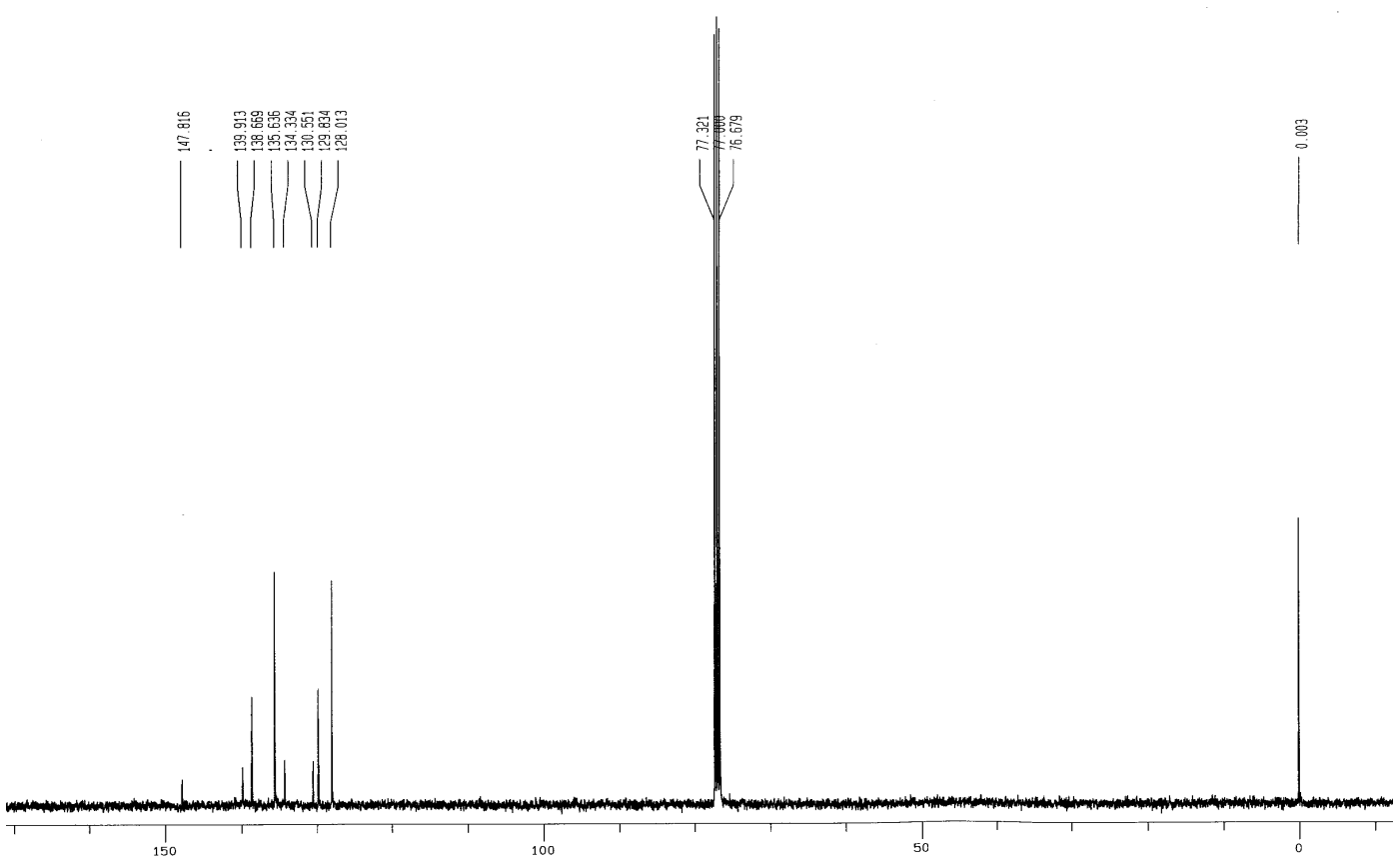

(b)

Figure 4. ${ }^{1} \mathrm{H}$ (a) and ${ }^{13} \mathrm{C}$ NMR spectra (b) of compound 5. 


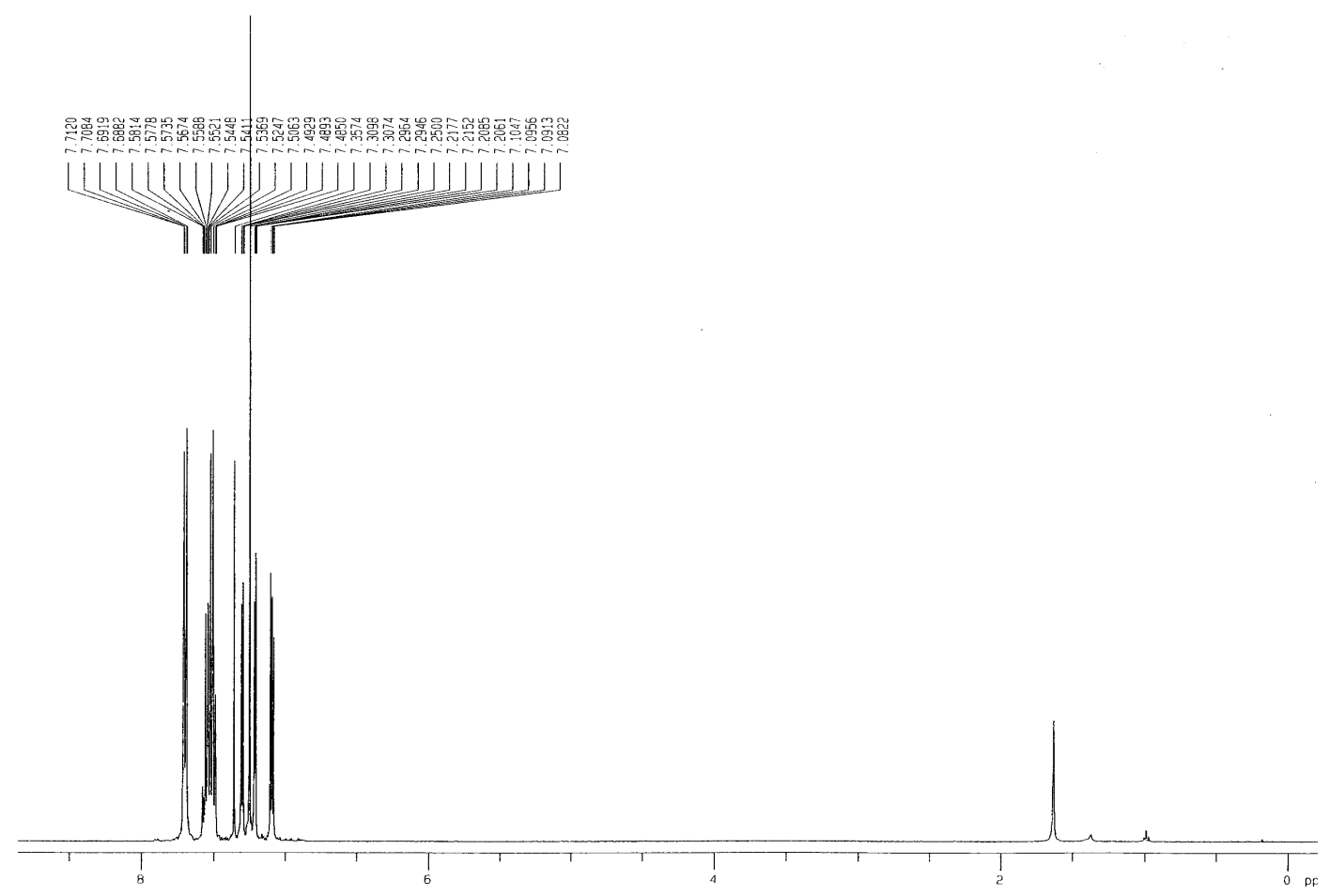

(a)

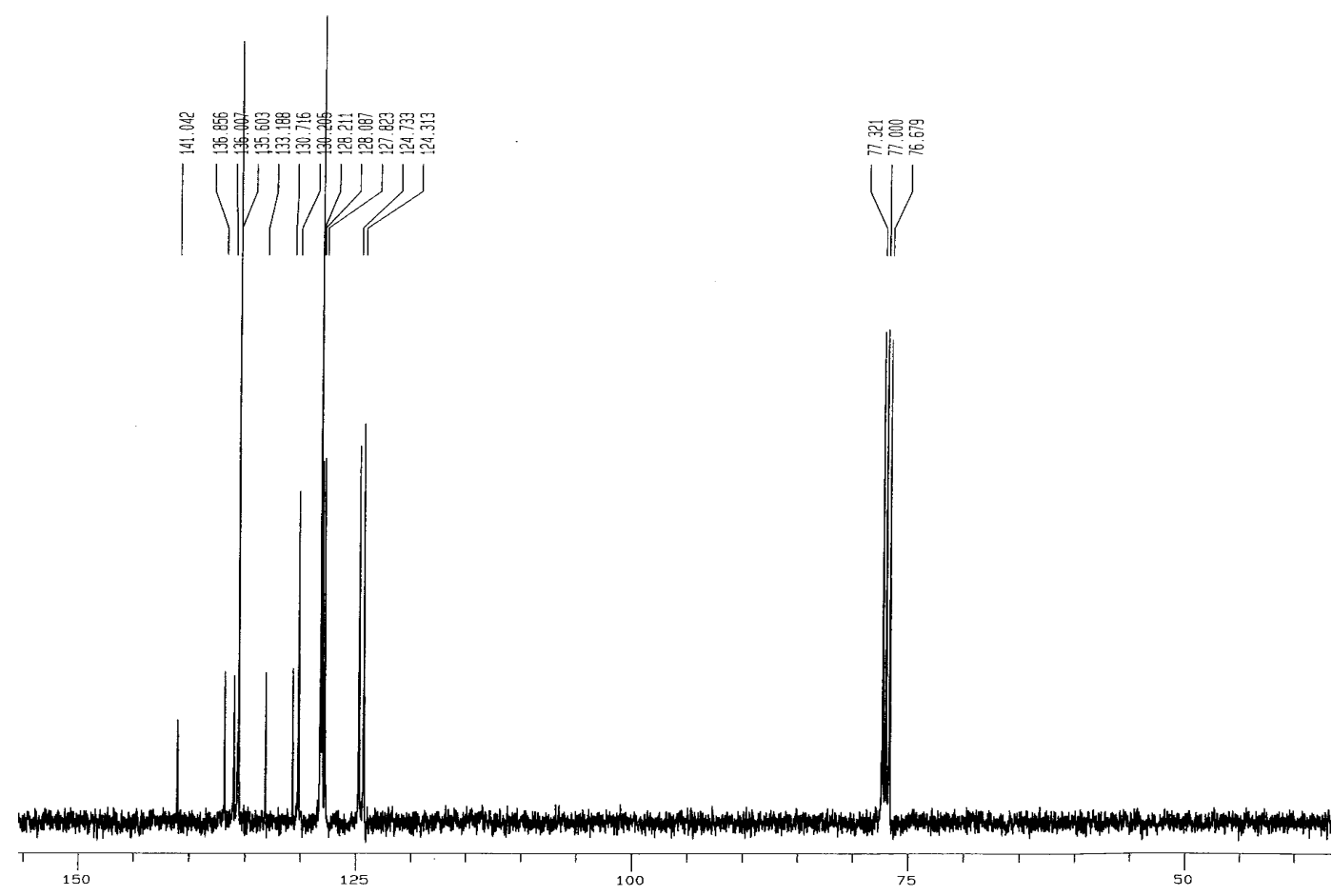

(b)

Figure 5. ${ }^{1} \mathrm{H}$ (a) and ${ }^{13} \mathrm{C}$ NMR spectra (b) of compound 11. 